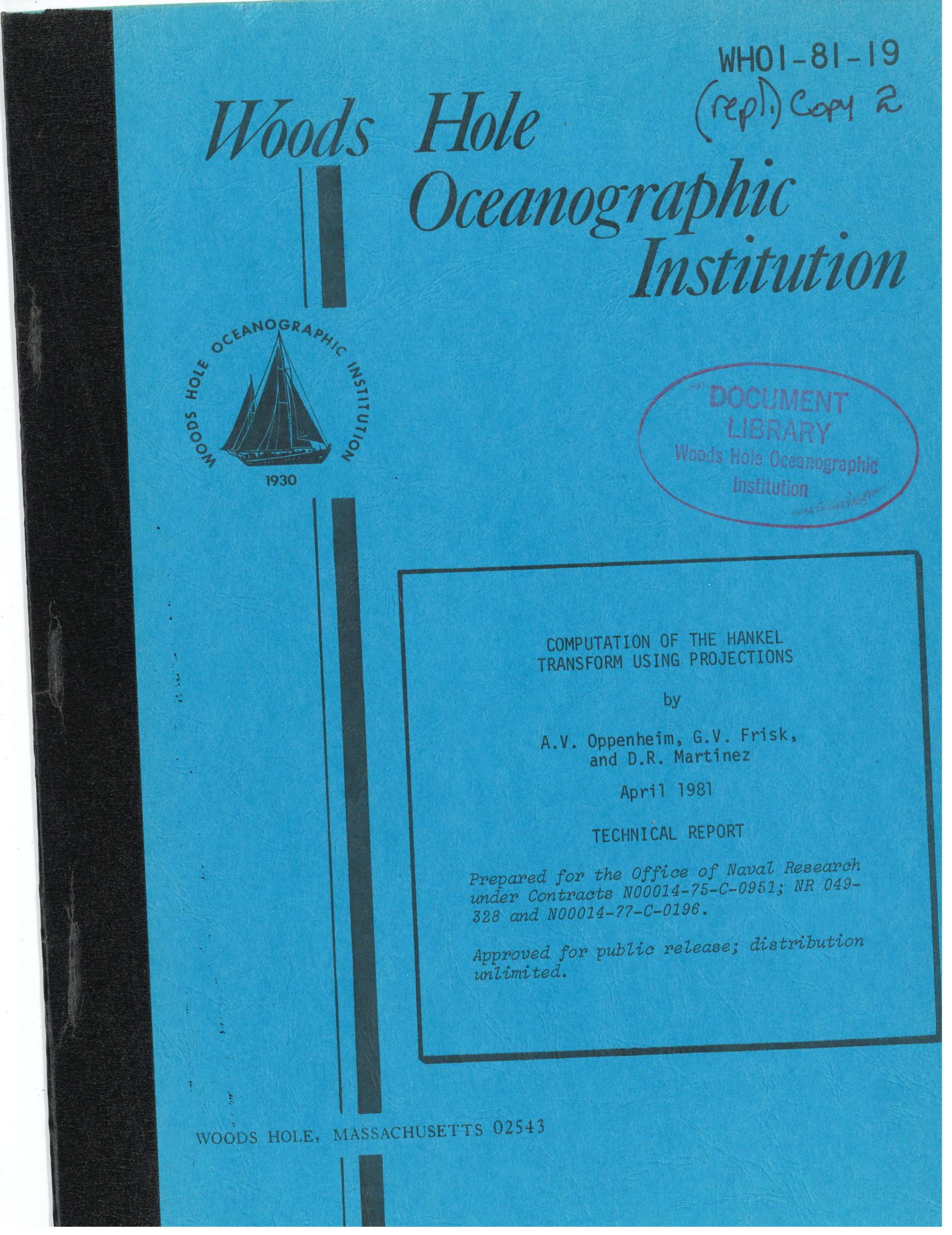


WHOI $-81-19$

COMPUTATION OF THE HANKEL

TRANSFORM USING PROJECTIONS

A.V. Oppenheim, G.V. Frisk,

by

D.R. Martinez

WOODS HOLE OCEANOGRAPHIC INSTITUTION

Woods Hole, Massachusetts 02543

Apri1 1981

TECHNICAL REPORT

Prepared for the Office of Naval Research under Contracts N00014-75-C-0951; NR 049-328 and N00014-77-C-0196.

Reproduction in whole or in part is permitted for any purpose of the United States Goverment. In citing this report in a bibliography, the reference given should be to: J. Acoust. Soc. Am. 68 (2): 523-529. (August 1980).

Approved for public release; distribution unlimited.

Approved for Distribution: Earl Hays, Chairman Department of Ocean Engineering 


\section{Computation of the Hankel transform using projections}

Alan V. Oppenheim

Research Laboratory of Electronics, Massachusetts Institute of Technology, Cambridge, Massachusetts 02139

George V. Frisk

Woods Hole Oceanographic Institution, Woods Hole, Massachusetts 02543

\section{David R. Martinez ${ }^{\text {a) }}$}

MIT/WHOI Joint Program, Oceanography/Oceanographic Engineering, Woods Hole, Massachusetts 02543 (Received 27 August 1979; accepted for publication 9 May 1980)

In this paper two new algorithms for computing an $n$ th-order Hankel transform are proposed. The algorithms are based on characterizing a circularly symmetric function and its two-dimensional Fourier transform by a radial section and interpreting the Hankel transform as the relationship between the radial section in the two domains. By utilizing the property that the projection of a two-dimensional function in one domain transforms to a radial section in the two-dimensional Fourier transform or inverse Fourier transform domain, several efficient procedures for computing the Hankel transform exploiting the one-dimensional FFT algorithm are suggested.

PACS numbers: 43.60.Gk, 43.30.Dr, 43.20.Fn, 02.30.Qy

\section{INTRODUCTION}

The need for numerical computation of the Hankel or Fourier-Bessel transform naturally arises in a variety of applications including optics, acoustics, electromagnetics and molecular biology. ${ }^{1-3}$ Most typically the Hankel transform arises as a consequence of the twodimensional Fourier transform of circularly symmetric functions. For example, for a horizontally stratified ocean bottom illuminated by an acoustic point source, the plane-wave reflection coefficient and the reflected pressure field are circularly symmetric and related through a two-dimensional Fourier transform. Applying the Fourier transform to the measured field, the plane-wave reflection coefficient can thus be calculated. Because of the circular symmetry, both the data and its Fourier transform can be specified in terms of a radial section or slice and the relationship between the radial section in the two domains is the Hankel transform.

There are a variety of procedures that have been proposed for computing a Hankel transform, taking advantage of the highly efficient FFT algorithm for computing the Fourier transform. In Sec. I we review some of these procedures. In Sec. II and III we propose two new procedures. These new algorithms are based on utilizing the fact that the projection of a two-dimensional function in one domain transforms to a radial section in the two-dimensional Fourier transform or inverse Fourier transform domain. As we develop in Secs. II and III, this property, which we refer to as the "projection-slice" theorem for two-dimensional Fourier transforms, leads to a procedure for computing the Hankel transform which exploits the efficiency of the one-dimensional FFT algorithm, and which avoids a number of the difficulties inherent in other procedures. In Sec. IV we present several ex-

\footnotetext{
${ }^{a}$ Present address: Atlantic Richfield Company, Dallas, TX 75221.
}

amples, motivated by a consideration of problems in ocean acoustics.

\section{TECHNIQUES FOR COMPUTATION OF THE HANKEL TRANSFORM USING THE FFT ALGORITHM}

The Hankel transform is closely related to the Fourier transform and in fact is generally associated with the two-dimensional Fourier transform of a circularly symmetric function. Specifically, let $f(x, y)$ and $F(\mu, \nu)$ denote a two-dimensional function and its Fourier transform in Cartesian coordinates so that

$$
F(\mu, \nu)=\frac{1}{2 \pi} \int_{-\infty}^{+\infty} \int_{-\infty}^{+\infty} f(x, y) \exp (j \mu x) \exp (j \nu y) d x d y
$$

or, with $f(x, y)$ and $F(\mu, \nu)$ expressed in polar coordinates,

$$
\begin{aligned}
\mathcal{F}(\rho, \phi)= & \frac{1}{2 \pi} \int_{0}^{2 \pi} \int_{0}^{\infty} f(r, \theta) \\
& \times \exp \{j[\cos (\theta-\phi)] r \rho\} r d r d \theta,
\end{aligned}
$$

with $f(r, \theta)$ and $\mathcal{F}(\rho, \phi)$ denoting the two-dimensional function and its Fourier transform in polar coordinates, where $\theta$ is measured relative to the $x$ axis and $\phi$ is measured relative to the $\mu$ axis. If $f(r, \theta)$ is of the form

$$
f(r, \theta)=g(r) \exp (j m \theta),
$$

where $g(r)$ is in general a complex function in $r$, and $m$ is an integer, then (2) reduces to ${ }^{1}$

$$
\mathcal{F}(\rho, \phi)=(j)^{m} G(\rho) \exp (j m \phi),
$$

where

$$
G(\rho)=\int_{0}^{\infty} J_{m}(r \rho) g(r) r d r .
$$

The integral relationship of Eq. (5) corresponds to the Hankel transform of order $m$.

There are a variety of methods which have been pro- 
posed for numerically evaluating the Hankel transform as given in Eq. (5), utilizing the efficiency of the FFT algorithm. One common procedure is to utilize the asymptotic expansion of the Bessel function. For example, for $x \gg 1, m$

$$
J_{m}(x) \simeq(2 / \pi x)^{1 / 2} \cos (x-m \pi / 2-\pi / 4)
$$

so that Eq. (5) becomes

$$
\begin{aligned}
G(\rho) & \simeq \int_{0}^{\infty}\left(\frac{2}{\pi r \rho}\right)^{1 / 2} \cos \left(r \rho-\frac{m \pi}{2}-\frac{\pi}{4}\right) g(r) r d r \\
& =\left(\frac{2}{\pi \rho}\right)^{1 / 2} \int_{0}^{\infty} r^{-1 / 2} g(r) \cos \left(r \rho-\frac{m \pi}{2}-\frac{\pi}{4}\right) d r .
\end{aligned}
$$

The integral in Eq. (7) corresponds to the cosine Fourier transform of $r^{-1 / 2} g(r)$ and thus can be numerically evaluated using the fast Fourier transform algorithm. The result is, of course, only approximate because of the asymptotic expansion used for the Bessel function and because of the sampling and truncation in $r$ required by the FFT algorithm.

Another algorithm, proposed by Tsang et al. ${ }^{4}$ is based on rewriting Eq. (5) by defining a new function $A(\lambda)$ as the inverse Fourier transform of $g(r)$ times an attenuation factor, i.e.,

$$
\begin{aligned}
& g(r) \exp (\nu r)=\int_{-\infty}^{+\infty} A_{\nu}(\lambda) \exp (j 2 \pi \lambda r) d \lambda \\
& A_{\nu}(\lambda)=\int_{-\infty}^{+\infty} g(r) \exp (\nu r) \exp (-j 2 \pi \lambda r) d r .
\end{aligned}
$$

In terms of $A_{\nu}(\lambda)$, Eq. (5) then becomes

$$
G(\rho)=\int_{0}^{\infty} \int_{-\infty}^{+\infty} J_{m}(r \rho) A_{\nu}(\lambda) \exp [-(\nu-j 2 \pi \lambda) r] d \lambda d r
$$

or

$$
G(\rho)=\int_{-\infty}^{+\infty} A_{\nu}(\lambda) I_{\nu}(\lambda, \rho) d \lambda,
$$

where

$$
I_{\nu}(\lambda, \rho)=\int_{0}^{\infty} \exp [-(\nu-j 2 \pi \lambda) r] J_{m}(r \rho) r d r .
$$

The function $I_{\nu}(\lambda, \rho)$ can be expressed in an analytic form and the function $A_{\nu}(\lambda)$ can be obtained at discrete values of $\lambda$ from Eq. (8b) utilizing the FFT algorithm. The integration in Eq. (9) is then approximated by a summation for each value of $\rho$ at which the Hankel transform is to be determined.

A third algorithm utilizing the FFT has been proposed by Siegman. ${ }^{5}$ In this algorithm, Eq. (5) is converted to a correlation by a change of variables. Specifically, with

$$
r=r_{0} e^{\hat{r}} \rho=\rho_{0} e^{\hat{\rho}}
$$

where $r_{0}$ and $\rho_{0}$ are constants, Eq. (5) becomes

$$
G\left(\rho_{0} e^{\hat{\rho}}\right)=\int_{-\infty}^{\infty} J_{m}\left[r_{0} \rho_{0} \exp (\hat{r}+\hat{\rho})\right] g\left(r_{0} e^{\hat{r}}\right) r_{0}^{2} \exp (2 \hat{r}) d \hat{r} .
$$

Equation (11) is the cross correlation of $J_{m}\left[r_{0} \rho_{0} e^{x}\right]$ and $g\left(r_{0} e^{x}\right) r_{0} \exp (2 x)$ and can thus be evaluated using the FFT algorithm. Since the use of the FFT in this context requires equally spaced sampling of the functions to be correlated and provides equally spaced samples of the result, both $g(r)$ and $G(\rho)$ will be sampled with exponential spacing. Thus, as $r$ decreases, $g(r)$ must be available at decreasing sampling intervals, which is often a disadvantage.

The algorithms to be described in this paper also exploit the efficiency of the FFT and appear, at least in some situations, to have a number of advantages over the methods outlined above. The methods are, in principle, exact as compared with the method based on the asymptotic expansion of the Bessel function. Furthermore, one of the methods developed in Secs. II and III generates equally spaced samples of the Hankel transform, $G(\rho)$, and the other accepts equally spaced samples of $g(r)$, thus avoiding the exponential spacing inherent in the method in Ref. 5. In addition, it appears to be computationally more straightforward than the method proposed in Ref. 4.

As developed in more detail in the next section, our proposed method of numerically evaluating (5) is based on a property of two-dimensional Fourier transforms which we refer to as the "projection-slice" theorem. 6,7 In essence, this theorem states that the one-dimensional transform of a projection of a two-dimensional function $f(x, y)$ at any angle is a radial section or slice at the same angle of its two-dimensional Fourier transform $F(\mu, \nu)$. Thus, for example, by using $g(r)$ to first compute a projection of $f(x, y)$ the one-dimensional FFT can be applied to obtain samples of $G(p)$. Alternatively, the one-dimensional transform can first be applied to $g(r)$ to obtain a projection of $F(\mu, \nu)$ after which one of several possible reconstruction algorithms can be used to obtain $F(\mu, \nu)$ and thus $G(\rho)$ from this projection. In the following section, we consider these possibilities in more detail.

\section{THE PROJECTION-SLICE THEOREM FOR TWO- DIMENSIONAL FUNCTIONS}

Our method for evaluating the Hankel transform is based on the projection-slice theorem for the twodimensional Fourier transform. Referring to Eq. (1), let us consider the slice in $F(\mu, \nu)$ corresponding to $\nu=0$, or equivalently $\mathfrak{F}(\rho, \phi)$ for $\phi=0$. Then

$$
F(\mu, 0)=\frac{1}{2 \pi} \int_{-\infty}^{+\infty} \exp (j \mu x) p(x) d x,
$$

where

$$
p(x)=\int_{-\infty}^{+\infty} f(x, y) d y .
$$

The one-dimensional function $p(x)$ is defined as the projection of $f(x, y)$ onto the $x$ axis and $F(\mu, 0)$ is a slice of $F(\mu, \nu)$ along the $\mu$ axis. More generally, the onedimensional transform of the projection of $f(x, y)$ onto a line in the $x-y$ plane at any angle is a slice of $F(\mu, \nu)$ along a radial line in the $\mu-\nu$ plane at the same angle. Thus, from (12) and (4), we can write that

$$
G(\rho)=\frac{j^{-m}}{2 \pi} \int_{-\infty}^{+\infty} \exp (j \rho x) p(x) d x .
$$

Comparing (14) and (5) it follows that the mth order 
Hankel transform can be equivalently expressed (and calculated) as $j^{-m}$ times the one-dimensional Fourier transform of the projection $p(x)$. The two basic computational steps in evaluating (5) are then the evaluation of the projection $p(x)$ as given by (13) and the evaluation of the one-dimensional Fourier transform in Eq. (14).

An alternate approach is to use the projection-slice theorem in reverse, that is, with the slice considered in the $x-y$ plane and the projection in the $\mu-\nu$ plane. Specifically,

$$
f(x, y)=\frac{1}{2 \pi} \int_{-\infty}^{+\infty} \int_{-\infty}^{+\infty} F(\mu, \nu) \exp (-j \mu) \exp (-j \nu y) d x d y,
$$

so that

$$
f(x, 0)=\frac{1}{2 \pi} \int_{-\infty}^{+\infty} \exp (-j \mu x) P(\mu) d \mu,
$$

where

$$
P(\mu)=\int_{-\infty}^{+\infty} F(\mu, \nu) d \nu .
$$

Equations (16) and (17) are the counterparts of Eqs. (12) and (13) and state that a slice along the $x$ axis of $f(x, y)$ is the inverse Fourier transform of a projection of $F(\mu, \nu)$ onto the $\mu$ axis.

As with Eqs. (12) and (13), this generalizes to a slice and projection at any angle. Now consider Eq. (2) rewritten as

$$
\mathcal{F}(\rho, \phi)=\frac{1}{2 \pi} \int_{0}^{2 \pi} d \theta \int_{0}^{\infty} f(r, \theta) \exp \{j[\cos (\theta-\phi) r \rho]\} r d r .
$$

The inner integral can be interpreted for each $\theta$ as the Fourier transform of the product of a slice, $f(r, \theta)$, at angle $\theta$ and the function $r u(r)$, where $u(r)$ is a unit step. Thus, the inner integral can be expressed as a convolution of the transforms of each of the terms in the product, i.e.,

$$
w[\rho \cos (\theta-\phi)] \triangleq \int_{0}^{\infty} f(r, \theta) \exp \{j[\cos (\theta-\phi) r \rho]\} r d r
$$

where

$$
\begin{aligned}
& w(\eta)=\int_{-\infty}^{\infty} P(\alpha, \theta) H(\eta-\alpha) d \alpha, \\
& P(\alpha, \theta)=\int_{-\infty}^{+\infty} f(r, \theta) \exp (j r \alpha) d r, \\
& H(\alpha)=\int_{0}^{\infty} r \exp (j r \alpha) d r .
\end{aligned}
$$

Equation (18) then becomes

$$
\mathcal{F}(\rho, \phi)=\frac{1}{2 \pi} \int_{0}^{2 \pi} w[\rho \cos (\theta-\phi)] d \theta .
$$

From Eqs. (3) and (4), to obtain the Hankel transform we take

$$
f(r, \theta)=g(r) \exp (j m \theta)
$$

and are interested in $\mathcal{F}(\rho, \phi)$ at $\phi=0$. In this case Eqs.
(19) and (20) lead to

$$
\begin{aligned}
& G(\rho)=j^{-m} \mathfrak{F}(\rho, 0)=\frac{j^{-m}}{2 \pi} \int_{0}^{2 \pi} w[\rho \cos \theta] \exp (j m \theta) d \theta, \\
& w(\eta)=\int_{-\infty}^{+\infty} P(\alpha) H(\eta-\alpha) d \alpha, \\
& P(\xi)=\int_{-\infty}^{+\infty} g(r) \exp (j r \xi) d r \\
& H(\xi)=\int_{0}^{+\infty} r \exp (j r \xi) d r .
\end{aligned}
$$

Equations (21) have a relatively straightforward interpretation in terms of the processing involved. Equation (21c) represents the Fourier transform of $g(r)$ which, according to Eq. (21b) is filtered (convolved) with a kernel $H(\xi)$. Equation (21a) then represents an operation referred to as back projection. Specifically, the integration in Eq. (21a) can be viewed in the following terms: For each $\theta$ we form the two-dimensional function $w(\rho \cos \theta) \exp (j m \theta)$ with $\rho$ varying along a line at an angle $\theta$ in the $\mu-\nu$ plane. This is referred to as a back projection of the function $w[\rho \cos \theta] \exp (j m \theta)$. All of these back projections are then superimposed to obtain $G(\rho)$.

Filtering of the projection, as specified in Eq. (21b), requires the kernel $H(\xi)$ which represents the impulse response of the filter. From Eq. (21d) it follows that this is in effect, a differentiator for which the impulse response formally does not converge. However, in any practical case, $G(\rho)$ will be assumed to be of finite extent so that $P(\xi)$ will be bandlimited.

The above theoretical discussion offers two alternative procedures for exploiting the projection-slice theorem for the two-dimensional Fourier transform in computing the Hankel transform. ${ }^{8}$ In the first, the function $g(r)$ is used to compute the projection in the $x-y$ plane which is then Fourier transformed to obtain a slice in the $\mu-\nu$ plane. In the second the function $g(r)$ is first Fourier transformed to obtain a projection in the $\mu-\nu$ plane. This projection is then filtered, followed by back projection according to Eq. (21a).

Thus far, in our work, we have only explored in detail the first of these possibilities. In the next section we consider some of the computational considerations associated with that specific procedure and in Sec. IV we present some examples.

\section{COMPUTATIONAL CONSIDERATIONS IN THE EVALUATION OF THE HANKEL TRANSFORM}

In this section we consider in more detail the evaluation of Eq. (5) using Eqs. (13) and (14).

The two basic computational steps in evaluating (5) using this approach are the evaluation of the projection $p(x)$ and the evaluation of the one-dimensional Fourier transform. Let us assume that $G(\rho)=0,|\rho|>R_{0}$. Then, from $(13) p(x)$ is bandlimited, and consequently, by virtue of the sampling theorem,

$$
j^{m} G(\rho)=\frac{\Delta x}{2 \pi} \sum_{k=-\infty}^{+\infty} p(k \Delta x) \exp (j \rho k \Delta x),
$$


provided that $\Delta x<\pi / R_{0}$. If we consider calculating $G(\rho)$ at $N$ equally spaced values $\Delta \rho=(1 / N)(2 \pi / \Delta x)$, then

$$
j^{m} G(k \Delta \rho)=\frac{\Delta x}{2 \pi} \sum_{n=0}^{N-1}\left\{\sum_{r=-\infty}^{\infty} p[(n+r N) \Delta x]\right\} \exp [j(2 \pi / N) n k] .
$$

Thus, $G(k \Delta \rho), k=0,1, \ldots, N-1$, is proportional to the discrete Fourier transform of the samples of $p(x)$, aliased in $x$. If the samples of $p(x)$ represent a finitelength sequence of length $\leqslant(N \Delta x)$, then (23) reduces to

$$
j^{m} G(k \Delta \rho)=\frac{\Delta x}{2 \pi} \sum_{n=0}^{N-1} p(n \Delta x) \exp \left(j \frac{2 \pi}{N} n k\right) .
$$

Both (23) and (24) correspond to the discrete Fourier transform, and consequently they can be evaluated directly using the one-dimensional FFT.

The calculation of samples of $p(x)$ is somewhat less direct. Equation (13) can equivalently be written as

$$
\begin{aligned}
& p(x)=2 \int_{0}^{+\infty} g\left[\left(x^{2}+y^{2}\right)^{1 / 2}\right] V_{m}\left(\frac{x}{\left(x^{2}+y^{2}\right)^{1 / 2}}\right) d y, \\
& p(x)=2 \int_{|x|}^{+\infty} g(r) \frac{r}{\left(r^{2}-x^{2}\right)^{1 / 2}} V_{m}\left(\frac{x}{r}\right) d r, \\
& p(x)=2|x| \int_{0}^{\pi / 2} g\left(\frac{x}{\cos \theta}\right) \frac{\cos m \theta}{\cos ^{2} \theta} d \theta,
\end{aligned}
$$

where $V_{m}(\cdot)$ is the mth-order Chebyshev polynomial. Equation (25) incorporates the fact that since $f(r, \theta)$ is circularly symmetric in $r$ and conjugate antisymmetric in $\theta$, only its even part contributes to $p(x)$. As indicated in Eq. (24), we wish to calculate equally spaced samples of the projection $p(x)$. If $g(r)$ is bandlimited, this is most easily done through the use of Eq. (25a). Specifically with $g(r)$ and hence $f(x, y)$ bandlimited,

$$
\int_{-\infty}^{+\infty} f(x, y) d y=\Delta y \sum_{k=-\infty}^{+\infty} f(x, k \Delta y),
$$

provided only that $\Delta y<2 \pi / R_{0}$. Equation (26) is basically a consequence of the fact that for a bandlimited function sampled at one-half the Nyquist rate or higher, its integral is directly proportional to the sum of its samples. Thus, $p(n \Delta x)$ as required in (23) or (24) is

$$
\begin{aligned}
p(n \Delta x)= & \Delta y \sum_{k=-\infty}^{+\infty} g\left\{\left[(n \Delta x)^{2}+(k \Delta y)^{2}\right]^{1 / 2}\right\} \\
& \times V_{m}\left(\frac{n \Delta x}{\left(n^{2} \Delta x^{2}+k^{2} \Delta y^{2}\right)^{1 / 2}}\right) .
\end{aligned}
$$

Equations (23) and (27) together provide an exact expression for the numerical calculation of $G(k \Delta \rho)$ provided only that $G(\rho)=0,|\rho|>R_{0}$. If this is not the case, then (24) will compute samples of $G(\rho)$ aliased in $\rho$, i.e.,

$$
\sum_{q=-\infty}^{+\infty} G[\Delta \rho(k+q N)]
$$

and an integration rule more complex than (26) must be used to calculate $p(x)$.

To evaluate (27) we assume that $g\left[\left(x^{2}+y^{2}\right)^{1 / 2}\right]$ is known on a rectangular grid in the $x-y$ plane. If $g(r)$ is only available as samples in $r$, then evaluation of (27) requires interpolation onto a rectangular grid.

\section{EXAMPLES}

In this section we present several examples of the computation of the zeroth-order Hankel transform using the algorithm outlined in the previous sections. A flow chart for the program used is given in the Appendix. In this program and in the examples $g(r)$ is assumed known as a function of the continuous variable $r$. To compute $G(\rho), g(r)$ was multiplied by a Hanning window $w(r)$ of the form

$$
w(r)= \begin{cases}0.5+0.5 \cos \left(\pi r / r_{0}\right), & 0 \leqslant r \leqslant r_{0} \\ 0, & \text { otherwise. }\end{cases}
$$

The sequence $s[n]$ corresponding to samples of the projection $p(x)$ of $g(r) w(r)$ was computed as

$$
s[n]=p\left[\left(n+\frac{1}{2}\right) \Delta x\right] .
$$

This spacing of samples was chosen so that an even sequence with an even number of points would result. Similarly, the spacing in $y$ was $2 \Delta x$, consistent with the discussion in the previous section. Samples of $G(\rho)$ were then obtained by applying the fast Fourier transform algorithm to $s[n]$.

\section{Example 1}

$$
g(r)= \begin{cases}1.0, & 0 \leqslant r \leqslant 1.0 \\ 0.0, & 1.0<r\end{cases}
$$
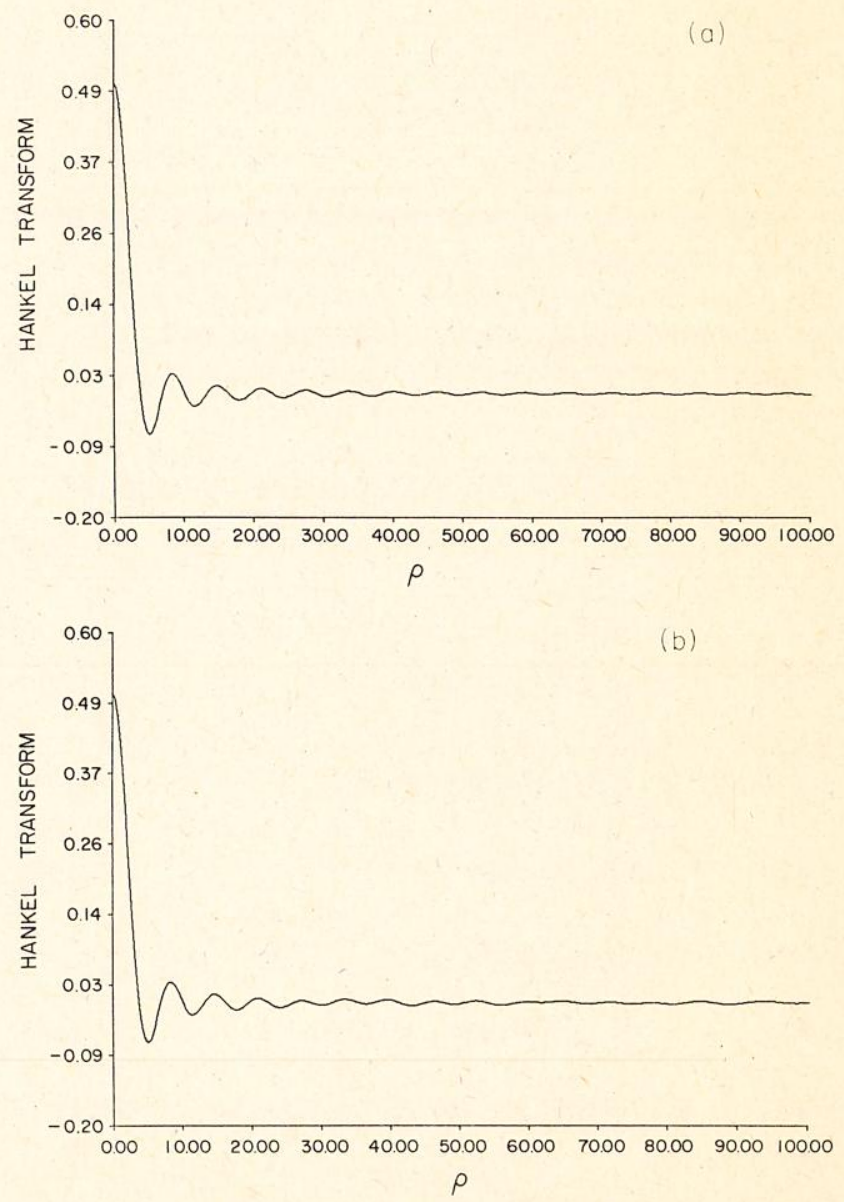

FIG. 1. Exact (a) and computed (b) Hankel transforms for example 1. 

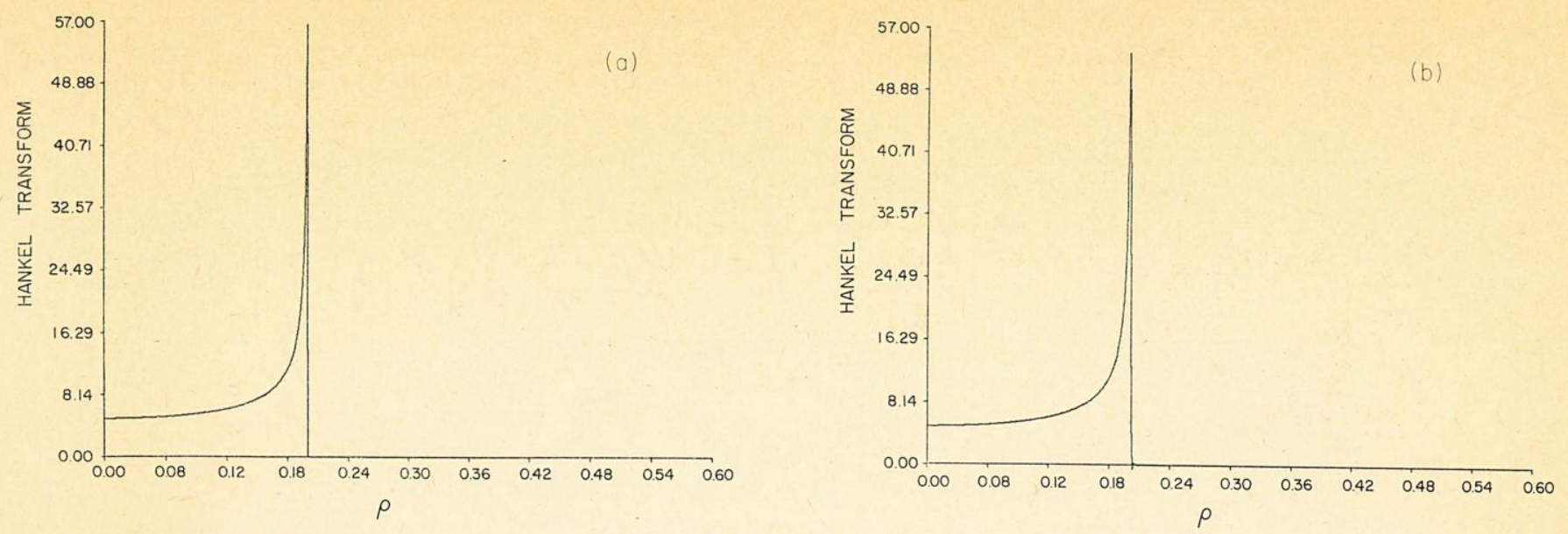

FIG. 2. Exact (a) and computed (b) Hankel transforms for example 2.

$$
\begin{aligned}
& G(\rho)=J_{1}(\rho) / \rho \\
& \Delta x=\pi / R_{0}=\pi / 100, \quad \Delta y=2 \Delta x, \quad r_{0}=2.0 .
\end{aligned}
$$

Figure 1(a) corresponds to $G(\rho)$ calculated directly from Eq. (30b) and Fig. $1(\mathrm{~b})$ to $G(\rho)$ as obtained by applying the Hankel transform algorithm to $g(r)$. As we see, there is excellent agreement between the results in Figs. 1(a) and 1(b).
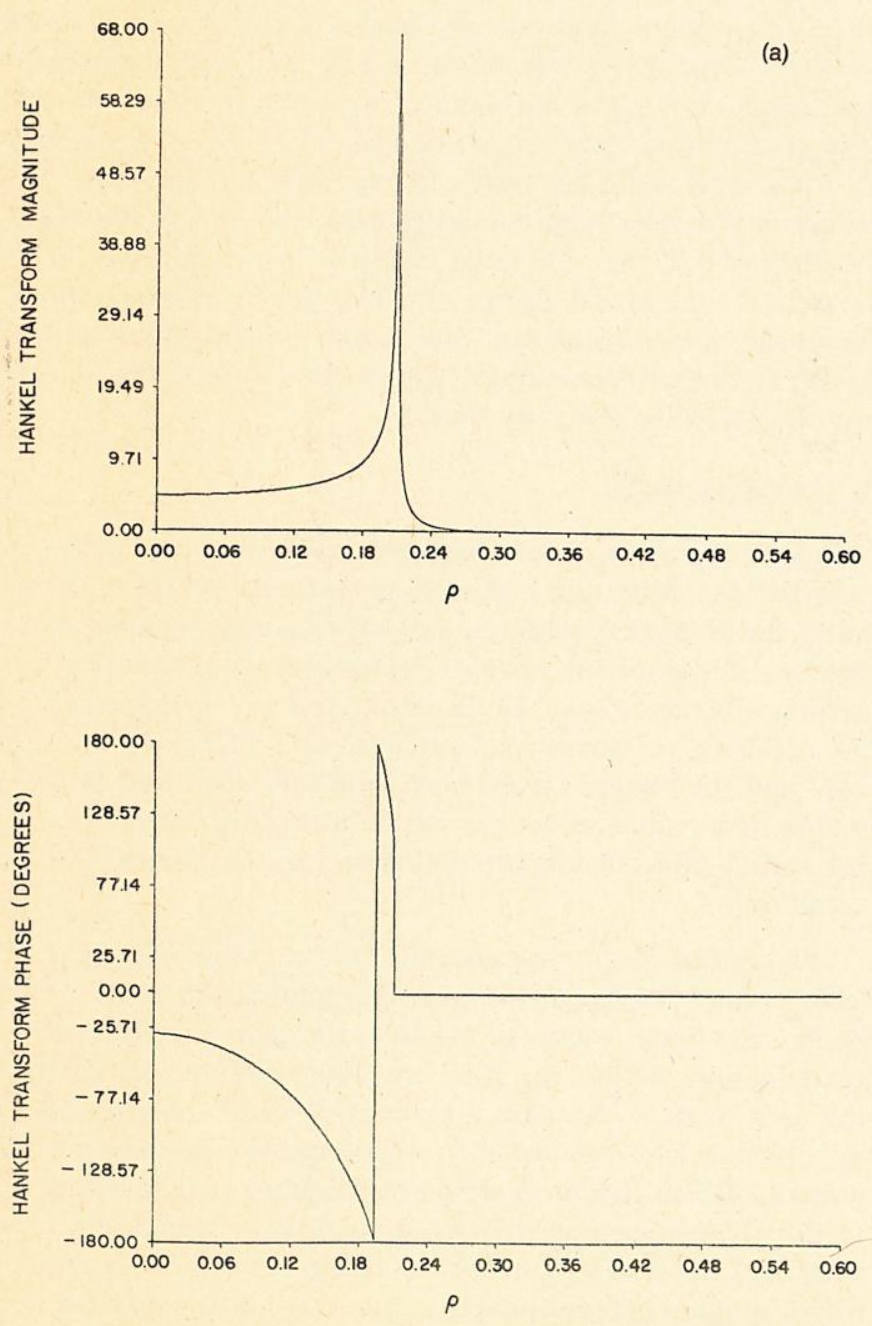

Example 2

$$
\begin{aligned}
& g(r)=\sin (0.2 r) / r . \\
& G(\rho)= \begin{cases}\frac{1}{\left[(0.2)^{2}-\rho^{2}\right]^{1 / 2}}, & 0<\rho \leqslant 0.2, \\
0, & 0.2<\rho,\end{cases} \\
& \Delta x=\pi / 0.6, \quad \Delta y=2 \Delta x, \quad r_{0}=2678.2 \text {. }
\end{aligned}
$$
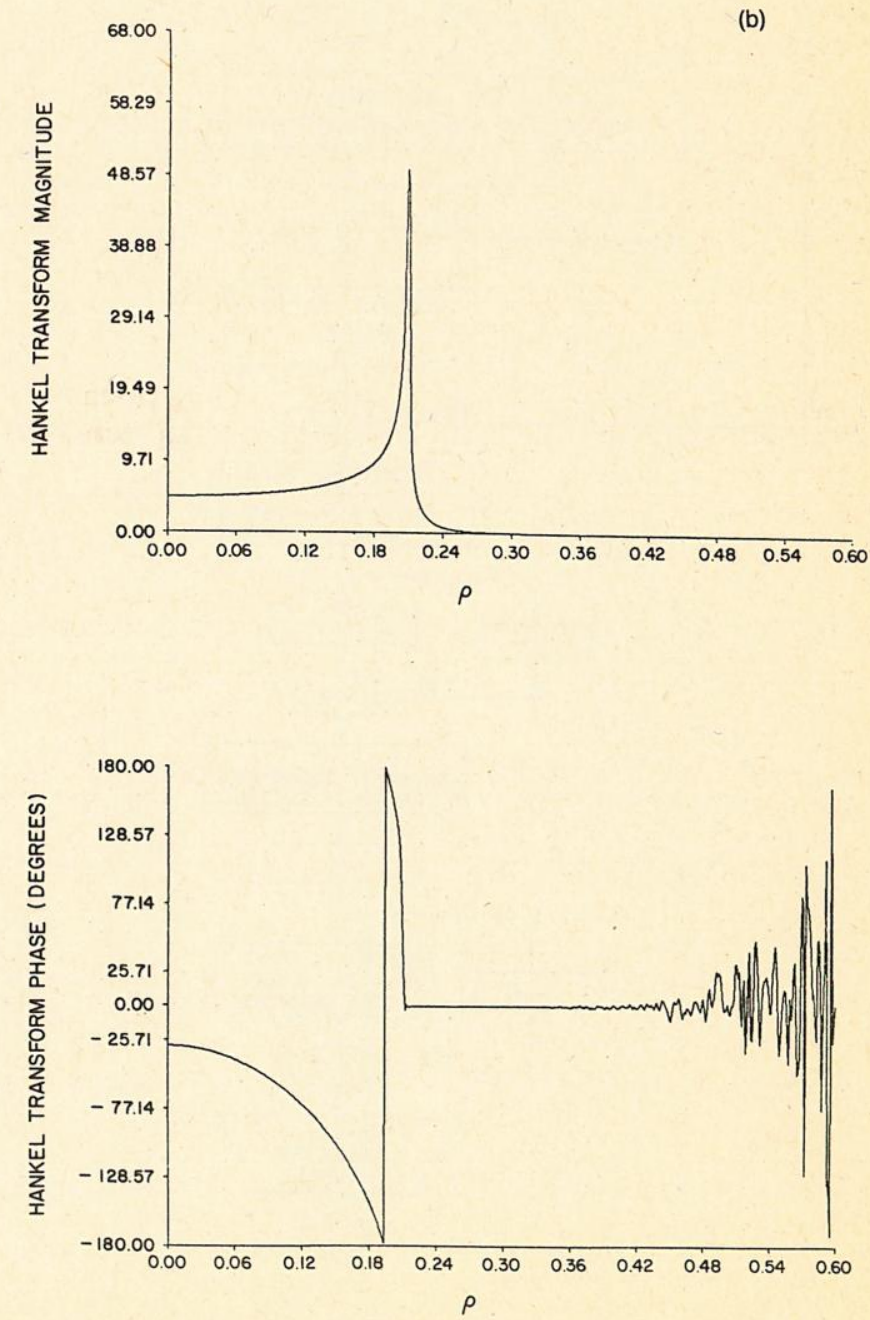

FIG. 3. Magnitude and phase of the exact (a) and computed (b) Hankel transforms for example 3. 


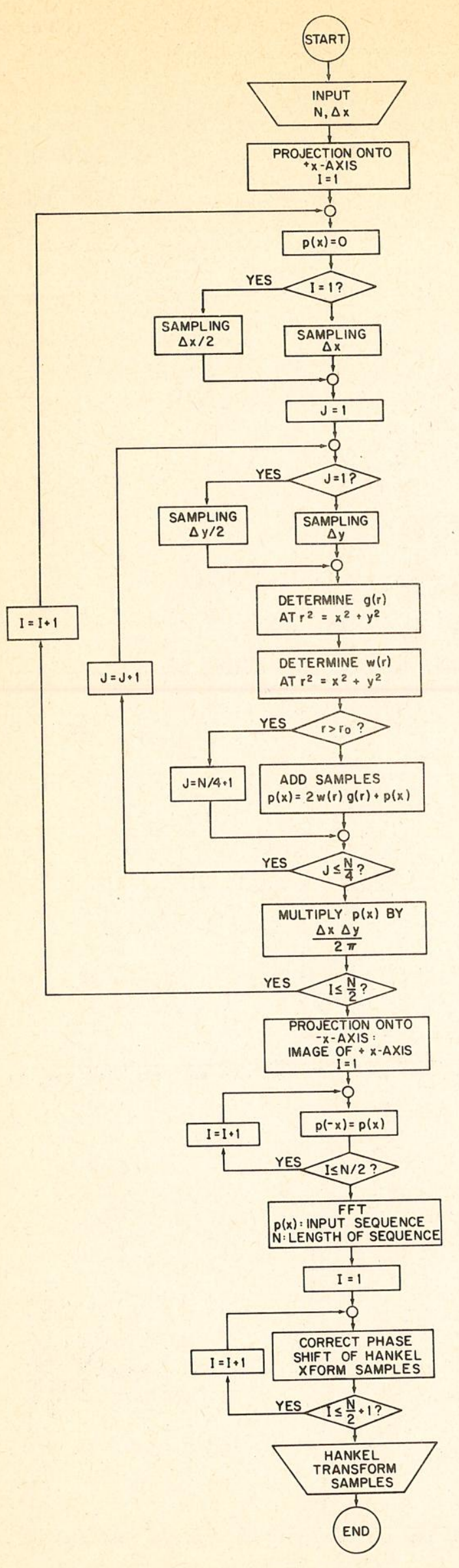

FIG. 4. Flow chart for the Hankel transform program.
This example was chosen because $g(r)$ is exactly bandlimited and thus $g(r)$ will be of infinite extent, requiring its truncation with a finite extent window. In addition it has a singularity in the Fourier domain that should accentuate the effects in $G(\rho)$ due to the window applied to $g(r)$. The results are shown in Fig. 2 with Fig. $2(a)$ corresponding to $G(\rho)$ as computed directly from Eq. (31b) and Fig. 2(b) to $G(\rho)$ as obtained by applying the Hankel transform algorithm to $g(r)$.

Example 3

$$
\begin{aligned}
& g(r)=\frac{\exp \left[j k_{0}\left(r^{2}+\delta^{2}\right)^{1 / 2}\right]}{\left(r^{2}+\delta^{2}\right)^{1 / 2}} . \\
& G(\rho)=j \frac{\exp \left[j \delta\left(k_{0}^{2}-\rho^{2}\right)^{1 / 2}\right]}{\left(k_{0}^{2}-\rho^{2}\right)^{1 / 2}} \\
& \Delta x=\pi / 0.6, \quad \Delta y=2 \Delta x, \quad r_{0}=2678.2, \\
& k_{0}=\frac{2 \pi \times 50}{1500} y, \quad \delta=20 .
\end{aligned}
$$

This example is associated with ocean acoustics and arises in the relationship between the acoustic pressure field and the reflection coefficient for a constant velocity water column and a perfectly reflecting ocean bottom. This example, in the context of ocean acoustics, is developed in considerable detail in the companion paper by Frisk, Oppenheim, and Martinez. ${ }^{9}$ The results with the choice of parameters above are shown in Fig. 3, with magnitude and phase of $G(\rho)$ as computed directly from Eq. (32b) shown in Fig. 3(a) and magnitude and phase of $G(\rho)$ as obtained by applying the Hankel transform algorithm to $g(r)$ in Fig. 3(b). As is evident in Figs. 3(a) and 3(b), there is excellent agreement between the exact and computed results, except for the oscillations in the computed phase as the magnitude becomes very small (large $\rho$ ). In general, it would be expected in any algorithm that phase computations will be highly sensitive numerically as the value of the magnitude becomes very small.

\section{v. CONCLUSION}

In this paper we have proposed two alternative procedures in computing a Hankel transform. Most typically the Hankel transform arises as a consequence of the two-dimensional Fourier transform of circularly symmetric functions. Because of circular symmetry, the methods proposed exploited the fact that both the data and its Fourier transform could be specified in terms of a radial slice and the relationship between the radial slice in the two domains was the Hankel transform.

This radial slice representation of a circularly symmetric function led directly to the application of the projection-slice theorem for two-dimensional Fourier transforms. Thus, the first method consisted of using the data to first compute a projection of the circularly symmetric function and then applying the one-dimensional FFT to obtain samples of the Hankel transform. In the second method the one-dimensional transform was first applied to the data to obtain a projection after which a back-projection algorithm was used to obtain the Hankel transform. 


\section{ACKNOWLEDGMENTS}

This work was supported in part by the Advanced Research Projects Agency, monitored by ONR under contract N00014-75-C-0951-NR049-328, at RLE, and in part by ONR contract N00014-77-C-0196, at WHOI. This paper is WHOI Contribution No. 4427.

\section{APPENDIX}

A flow chart for the program used to compute the zeroth-order Hankel transform of $g(r)$ is illustrated in Fig. 4.

A description of the function and variables follows:

$N$ number of samples; an integer power of two

$\Delta x \quad$ sampling interval in $x$

$p(x)$ projection onto $x$ axis

$\Delta y \quad \Delta x / 2$

$g(r)$ circularly symmetric function

$w(r)$ circularly symmetric window

In the evaluation of the projection, $g(r)$ is assumed known on a two-dimensional grid as a function of the continuous variable $r=\left(x^{2}+y^{2}\right)^{1 / 2}$. Only the first quadrant must be sampled since the function is circularly symmetric. The projection samples onto the negative $x$ axis are simply found by forming the image of the positive $x$ axis. These equally spaced samples are then used as the input to a one-dimensional FFT. A phase shift correction to the output samples must be made since the sequence as the input to the FFT is interpreted starting at the origin.

${ }^{1}$ A. Papoulis, Systems and Transforms with Applications in Optics (McGraw-Hill, New York, 1968).

${ }^{2}$ W. M. Ewing, W. S. Jardetzky, and F. Press, Elastic Waves in Layered Media (McGraw-Hill, New York, 1957).

${ }^{3}$ D. J. DeRosier and A. Klug, "Reconstruction of Three-Dimensional Structures from Electron Micrographs," Nature 217, 130-134 (1968).

${ }^{4}$ L. Tsang, R. Brown, J. A. Kong, and G. Simmons, "Numerical Evaluation of Electromagnetic Fields Due to Dipole Antennas in the Presence of Stratified Media," J. Geophys. Res. 79, 2077-2080 (1974).

${ }^{5}$ A. E. Siegman, "Quasi Fast Hankel Transform," Opt. Lett. 1, 13-15 (July 1977).

${ }^{6}$ R. N. Bracewell, "Strip Integration in Radio Astronomy," Aust. J. Phys. 9, 198-217 (1956).

${ }^{7}$ R. Mersereau and A. Oppenheim, "Digital Reconstruction of Multi-dimensional Signals from their Projections," Proc. IEEE, 62, 1319-1338 (1974).

${ }^{8}$ A. V. Oppenheim, G. V. Frisk, and D. R. Martinez, "An Algorithm for the Numerical Evaluation of the Hankel Transform," Proc. IEEE, 66, 264-265 (1978).

${ }^{9}$ G. V. Frisk, A. V. Oppenheim, and D. R. Martinez, "A Technique for Measuring the Plane Wave Reflection Coefficient of the Ocean Bottom, "J. Acoust. Soc. Am. 68, 602612 (1980). 
MANDATORY DISTRIBUTION LIST

FOR UNCLASSIFIED TECHNICAL REPORTS, REPRINTS, AND FINAL REPORTS PUBLISHED BY OCEANOGRAPHIC CONTRACTORS

OF THE OCEAN SCIENCE AND TECHNOLOGY DIVISION

OF THE OFFICE OF NAVAL RESEARCH

(REVISED NOVEMBER 1978)

1 Deputy Under Secretary of Defense

(Research and Advanced Technology)

Military Assistant for Environmental Science Room 3D129

Washington, D.C. 20301

Office of Naval Research

800 North Quincy Street

Arlington, VA 22217

3 ATTN: Code 483

1 ATTN: Code 460

2 ATTN: 102B

1 CDR J. C. Harlett, (USN)

ONR Representative

Woods Hole Oceanographic Inst.

Woods Hole, MA 02543

Commanding Officer

Naval Research Laboratory

Washington, D.C. 20375

6 ATTN: Library, Code 2627
12 Defense Documentation Center

Cameron Station

Alexandria, VA 22314

ATTN: DCA

Commander

Naval Oceanographic Office

NSTL Station

Bay St. Louis, MS 39522

1 ATTN: Code 8100

1 ATTN: Code 6000

1 ATTN: Code 3300

1 NODC/NOAA

Code D781

Wisconsin Avenue, N.W.

Washington, D.C. 20235 
SECURITY CLASSIFICATION OF THIS PAGE (When Data Entored)

\begin{tabular}{|c|c|c|}
\hline \multicolumn{2}{|r|}{ REPORT DOCUMENTATION PAGE } & $\begin{array}{l}\text { READ INSTRUCTIONS } \\
\text { BEFORE COMPLETING FORM }\end{array}$ \\
\hline & \begin{tabular}{l|l} 
REPORT NUMBER & 2. GOVT ACCESSION NO. \\
WHOI $-81-19$ &
\end{tabular} & 3. RECIPIENT'S CATALOG NUMBER \\
\hline \multirow{2}{*}{\multicolumn{2}{|c|}{$\begin{array}{l}\text { 4. TITLF (and Subtilla) } \\
\text { COMPUTATION OF THE HANKEL TRANSFORM USING } \\
\text { PROJECTIONS }\end{array}$}} & $\begin{array}{l}\text { 5. TYPF OF REPORT A PERIOD COVERED } \\
\text { Technical }\end{array}$ \\
\hline & & $\begin{array}{l}\text { 6. PERFORMING ORG. REPORT NUMBER } \\
\text { WHOI Cont. \#4427 }\end{array}$ \\
\hline \multicolumn{2}{|r|}{$\begin{array}{l}\text { A. AU THOR(a) Oppenheim, G.V. Frisk, and D.R. Martinez } \\
\text { A. }\end{array}$} & $\begin{array}{l}\text { 8. CONTRACT OR GRANT NUMBER( } \theta) \\
\text { N00014-75-C-0951; * } \\
\text { N00014-77-C-0196 }\end{array}$ \\
\hline \multicolumn{2}{|r|}{$\begin{array}{l}\text { 9. PERFORMING ORGANIZATION NAME AND ADDRESS } \\
\text { WOODS HOLE OCEANOGRAPHIC INSTITUTION } \\
\text { Woods Hole, Massachusetts } 02543\end{array}$} & $\begin{array}{l}\text { 10. PROGRAM ELEMENT, PROJECT, TASK } \\
\text { AREA Q WORK UNIT NUMBERS } \\
\text { * NR } 049-328\end{array}$ \\
\hline \multirow{2}{*}{\multicolumn{2}{|c|}{$\begin{array}{l}\text { 11. CONTROLLING OFFICE NAME AND ADDRESS } \\
\text { NORDA/National Space Technology Laboratory } \\
\text { Bay St. Louis, MS } 39529\end{array}$}} & $\begin{array}{|ll|}\text { 12. REPORT DATE } & \\
\text { April } & 1981 \\
\end{array}$ \\
\hline & & 13. NUMBER OF PAGES \\
\hline \multirow{2}{*}{\multicolumn{2}{|c|}{ 14. MONITORING AGENCY NAME \& ADDRESS(if difforent from Controlling Office) }} & $\begin{array}{l}\text { 15. SECURITY CLASS. (of thio roport) } \\
\text { Unclassified }\end{array}$ \\
\hline & & $\begin{array}{l}\text { 15a. DECLLASSIFICATION/DOWNGRADING } \\
\text { SCHEDULE }\end{array}$ \\
\hline
\end{tabular}

17. DISTRIBUTION STATEMENT (of the abetract ontered in Block 20, if difforent from Roport)

18. SUPPLEMENTARY NOTES

Reprinted from: J. Acoust. Soc. Am. 68 (2): 523-529. (August 1980),

19. KEY WORDS (Continue on roverse olde if noceseary and identify by block number)

1. Hankel transform

2. Projection-slice theorem

3. Fast Fourier transform

20. ABSTRACT (Continue on revereo olde if neceseary and identify by block number)

In this paper two new algorithms for computing an nth-order Hankel transform are proposed. The algorithms are based on characterizing a circularly symmetric function and its two-dimensional Fourier transform by a radial section and interpreting the Hankel transform as the relationship between the radial section in the two domains. By utilizing the property that the projection of a two-dimensional function in one domain transforms to a radial section in the two-dimensional Fourier transform or inverse Fourier transform domain, (cont. on back) 
UNCLASSIFIED $\quad 4 / 81$

- LCURITY CLASSIFICATION OF THIS PAGE(When Date Entered)

several efficient procedures for computing the Hanker transform exploiting the one-dimensional FFT algorithm are suggested.

UNCLASSIFIED 4/81 


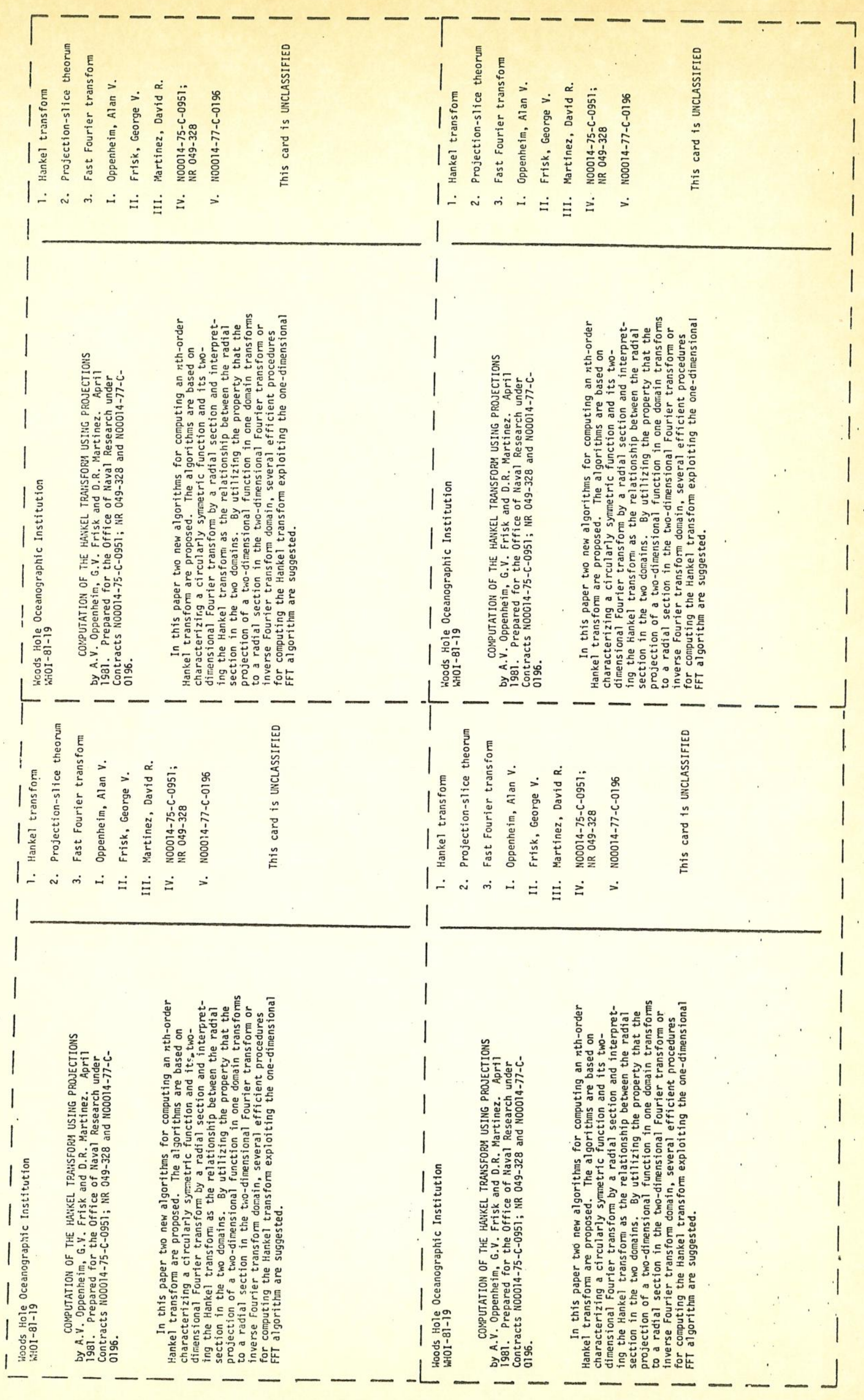

